\title{
Projeto ReFormar: Extensão em Engenharia Civil baseada nas Diretrizes para a Extensão Universitária
}

DOI: 10.37702/2175-957X.COBENGE.2021.3419

Yuri Mariano Carvalho - yuri.mariano@engenharia.ufff.br

Universidade Federal de Juiz de Fora

Rua Oswaldo Campos 25

36610-000 - Pequeri - MG

Mariana Oliveira de Castro - mariana.castro@engenharia.ufjf.br

Universidade Federal de Juiz de Fora

Rua Pedro Gerhein 69

36036-620 - Juiz de Fora - MG

Pedro Freguglia Roque - pedro.freguglia@engenharia.ufjf.br

Universidade Federal de Juiz de Fora

RUA PARACATU 1300

36047-040 - Juiz de Fora - MG

Rômulo Heleno Silva Bandeira - romulo.heleno@engenharia.ufjf.br

Universidade Federal de Juiz de Fora

Rua José Augusto de Abreu 429

36883-031 - Muriaé - MG

Yngrid de Paula Oliveira - yngrid.oliveira@engenharia.ufjf.br

Universidade Federal de Juiz de Fora

Presidente Costa e Silva 203

36140-000 - Lima Duarte - MG

Julia Righi de Almeida - julia.righi@engenharia.ufjf.br

Universidade Federal de Juiz de Fora

Rua Monsenhor Gustavo Freire 21

36016-470 - Juiz de Fora - MG

Resumo: Segundo as Diretrizes para a Extensão na Educação Superior Brasileira, a Extensão deve promover uma ação transformadora que conjugue Universidade e sociedade em uma interação dialógica, por meio do compartilhamento e da 
construção conjunta de conhecimentos. Todavia, realizar atividades de Extensão que promovam o desenvolvimento de competências entre os estudantes e ainda impactem positivamente a sociedade tem sido um desafio para os cursos de graduação. Nesse contexto, o Programa de Educação Tutorial do curso de Engenharia Civil da Universidade Federal de Juiz de Fora (PET Civil - UFJF) elaborou uma atividade de extensão pautada nas Diretrizes para a Extensão Universitária denominada Projeto ReFormar. O projeto objetiva oferecer auxílio técnico de Engenharia Civil a organizações sociais ou públicas a fim de conceber novos conhecimentos e produtos em conjunto com a comunidade envolvida na atividade. Este artigo sintetiza a motivação para o planejamento da atividade, as etapas pensadas para a sua execução e os resultados esperados com sua implementação. Dessa forma, espera-se atender às Diretrizes para a Extensão na Educação Superior Brasileira e contribuir para o desenvolvimento de atividades de extensão similares em outras Instituições de Ensino Superior.

Palavras-chave: Engenharia e sociedade. Transformação social. Tríade acadêmica. Assistencialismo. 


\section{INTRODUÇÃO}

Assegurada pelo Artigo $207^{\circ}$ da Constituição Federal (BRASIL, 1988), a Extensão será elemento obrigatório da grade curricular dos cursos de graduação brasileiros a partir de 2023 (BRASIL, 2020). No contexto das Engenharias, a curricularização da Extensão é reforçada pelas atuais Diretrizes Curriculares Nacionais do Curso de Graduação em Engenharia (DCNs de Engenharia). Como as atividades de extensão conjugam teoria e prática em contextos reais de aplicação (BRASIL, 2019), os estudantes engajados nessas atividades assumem o protagonismo de sua aprendizagem e desenvolvem competências essenciais para sua atuação profissional e cidadã. Todavia, desenvolver atividades de extensão que incentivem o desenvolvimento de competências e que promovam uma real transformação entre Universidade e sociedade ainda é um grande desafio.

Até o século XIX, a interlocução entre as Instituições de Ensino Superior (IES) e sociedade não perpassava pelos grandes problemas sociais da época, mas sim pelos anseios mercantis em prol de novas técnicas e tecnologias (MOREIRA, 2014). As primeiras iniciativas universitárias de capacitações e prestações de serviços à comunidade surgiram no Brasil no início do século XX, ainda com caráter predominantemente assistencialista, como aponta o Fórum de Pró-Reitores das Instituições Públicas de Educação Superior Brasileiras (FORPROEX). Somente com e após as lutas pela redemocratização é que a Extensão foi confirmada como instrumento de inter-relação entre Universidade e sociedade por meio da troca de saberes (FORPROEX, 2012). Logo, a Extensão deve ser encarada como uma via de mão dupla que promove tanto o aprendizado do extensionista (educadoreducando) quanto o aprendizado dos sujeitos da ação de extensão (educandoseducadores) (FREIRE, 1983) - o que vai de encontro aos minicursos abertos à população, às ações de caridade e às feiras de ciências promovidos por muitas instituições (MOREIRA, 2014).

A fim de promover uma Extensão verdadeiramente transformadora para Universidade e sociedade, o Programa de Educação Tutorial do curso de Engenharia Civil da Universidade Federal de Juiz de Fora (PET Civil - UFJF) propôs um projeto de extensão pautado na Resolução no 7, de 18 de dezembro de 2018, que institui as Diretrizes para a Extensão na Educação Superior Brasileira.

\section{REFERENCIAL TEÓRICO}

A Extensão está presente na matriz curricular dos cursos de graduação brasileiros integrada, usualmente, a atividades de ensino e pesquisa. Trata-se de um processo interdisciplinar de cunho político-educacional, cultural, científico e tecnológico que objetiva promover interações transformadoras entre Universidade e sociedade (BRASIL 2018). Essa interação transformadora se dá pela ação de um sujeito (individual ou coletivo) que se utiliza de seus conhecimentos e técnicas para agir sobre uma problemática considerando o contexto social em que ela se insere. Ao reconhecer esse contexto social, o extensionista é capaz de problematizar a homens e mulheres da comunidade a situação real em que vivem para que, entendendo-a criticamente, também possam atuar criticamente sobre ela (FREIRE, 1983).

Em 2012, o FORPROEX (2012) acordou diretrizes para orientar a formulação e implementação da Extensão nas IES brasileiras. Já em 2018, as atividades de extensão passaram a ser regulamentadas por uma resolução própria — as Diretrizes para a Extensão na Educação Superior Brasileira, as quais estão pautadas na interação transformadora 
entre Universidade e sociedade por meio da produção e da aplicação de conhecimentos. Essa interação deve estar fundamentada nos princípios explicitados nos Artigos $5^{\circ}$ e 6음 das Diretrizes para a Extensão Universitária (BRASIL, 2018), os quais foram sintetizados no Quadro 1, a seguir, sob a nomenclatura inicialmente proposta na PNEU (FORPROEX, 2012).

Quadro 1 - Síntese das Diretrizes para a Extensão na Educação Superior Brasileira

\begin{tabular}{|c|c|c|}
\hline DIRETRIZ & DESCRIÇÃO & $\begin{array}{c}\text { ALUSÕES NO } \\
\text { MARCO LEGAL }\end{array}$ \\
\hline Interação Dialógica & $\begin{array}{l}\text { Diálogo com comunidades, movimentos sociais e } \\
\text { setores do poder público e/ou privado em prol da } \\
\text { produção, troca e aplicação de conhecimentos, } \\
\text { reafirmando a dimensão ética da Extensão. }\end{array}$ & 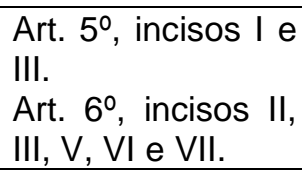 \\
\hline $\begin{array}{l}\text { Interdisc } \\
\text { Interprof }\end{array}$ & $\begin{array}{l}\text { Reflexão quanto à complexidade de uma realidade } \\
\text { social equilibrando visões holísticas e especializadas } \\
\text { advindas de diversas áreas do conhecimento. }\end{array}$ & $\begin{array}{l}\text { Art. 50, inciso II. } \\
\text { Art. 6o, incisos III, } \\
\text { V e VI. }\end{array}$ \\
\hline $\begin{array}{r}\text { Indissociak } \\
\text { Ensino-Pesquis }\end{array}$ & $\begin{array}{l}\text { Processo acadêmico que potencializa as atividades } \\
\text { de extensão ao vinculá-las à formação técnica e } \\
\text { cidadã dos estudantes (Ensino) e à geração de } \\
\text { conhecimentos a partir de metodologias participativas } \\
\text { (Pesquisa). }\end{array}$ & $\begin{array}{l}\text { Art. 5o, inciso IV. } \\
\text { Art. 6o, incisos II, } \\
\text { III, IV, V, VI e VII. }\end{array}$ \\
\hline $\begin{array}{r}\text { Impacto na For } \\
\text { Estudan }\end{array}$ & $\begin{array}{l}\text { Contribuição significativa à formação discente por } \\
\text { meio do contato direto com questões sociais } \\
\text { contemporâneas, reforçando, assim, os } \\
\text { compromissos éticos da Educação Superior } \\
\text { brasileira. }\end{array}$ & $\begin{array}{l}\text { Art. } 5^{\circ} \text {, inciso I e II. } \\
\text { Art. } 6^{\circ} \text {, incisos III, } \\
\text { V e VI. }\end{array}$ \\
\hline $\begin{array}{c}\text { Impacto e Transformação } \\
\text { Social }\end{array}$ & $\begin{array}{l}\text { Atuação transformadora que impacta tanto a } \\
\text { Universidade quanto a sociedade através do } \\
\text { desenvolvimento social e regional pautado nas reais } \\
\text { necessidades identificadas em uma comunidade, } \\
\text { reforçando o caráter político da Extensão. }\end{array}$ & $\begin{array}{l}\text { Art. } 5^{\circ} \text {, inciso I, II e } \\
\text { III. } \\
\text { Art. 6으, incisos I, II } \\
\text { III, IV, V, VI e VII. }\end{array}$ \\
\hline
\end{tabular}

Fonte: Adaptado de FORPROEX (2012) e Brasil (2018).

Para atender a tais diretrizes, os extensionistas devem estabelecer um diálogo com a comunidade que promova a construção conjunta e a troca e de saberes entre ambos, e não que apenas oferecer algo à comunidade - caracterizando, assim, ideais puramente messiânicos e assistencialistas. Uma atividade assistencialista ocorre quando seus agentes estendem o conhecimento produzido numa IES para a comunidade sem problematizar, junto a esta, o seu próprio contexto social.

Segundo Alayón (1992), o assistencialismo garante a "preservação do privilégio nas mãos de poucos", visto ser uma ação implementada para reduzir a miséria sem, necessariamente, tratar suas causas. Ao encarar a Extensão como uma transferência de conhecimentos ou tecnologias da "sede do saber" a fim de "salvar" aqueles que habitam na "sede da ignorância", nega-se a capacidade humana de transformar a própria realidade em que se insere. Logo, o assistencialismo não é dialógico em sua ação quando seus agentes se consideram os únicos agentes da mudança - visto desconsiderar os seres humanos no contexto da ação - o que contribui para a negação do indivíduo enquanto ser pensante por si próprio através de suas relações com o mundo (FREIRE, 1983). A fim de evitar que atividades assistencialistas sejam registradas como atividades de extensão, é importante que discentes, funcionários e docentes sejam receptivos a propostas advindas da própria sociedade, como aquelas advindas de movimentos sociais (MOREIRA, 2014) — contribuindo, dessa forma, para o cumprimento das Diretrizes para a Extensão na Educação Superior Brasileira. 
O cumprimento dessas diretrizes deverá ser avaliado pelas IES considerando a pertinência das atividades e extensão para a creditação curricular, a contribuição das atividades para o cumprimento dos objetivos institucionais e os resultados alcançados com as atividades. O Instituto Anísio Teixeira (INEP), por sua vez, será responsável pela avaliação externa in loco; a autarquia considerará em sua avaliação a previsão e o cumprimento da carga horária mínima para Extensão nos currículos dos cursos de graduação (10\% da carga horária total), a indissociabilidade Ensino-Pesquisa-Extensão e os docentes responsáveis pelas atividades de extensão (BRASIL, 2018). Considerando tais critérios, é válido desenvolver um instrumento que permita às universidades avaliar as atividades de extensão que estão desenvolvendo e ainda comparar o desempenho de suas atividades com as de outras IES sob os mesmos critérios.

Fazendo jus a essa necessidade por uma metodologia para avaliar a Extensão na Educação Superior Brasileira, Kienetz, Vieira e Visentini (2020) estruturaram um instrumento multidimensional de avaliação da Extensão após verificarem dados de 63 IES federais. Os autores identificaram que a maioria das universidades analisadas possui resolução que regulamenta as atividades de extensão $(86 \%)$ e fomento para 0 desenvolvimento destas (92\%), mas que a avaliação dessas atividades está condicionada, principalmente, aos editais de submissão de propostas (73\%) - havendo somente 6 IES que possuem critérios de pontuação para o registro da atividade de extensão. Nesse contexto, o instrumento proposto por Kienetz, Vieira e Visentini (2020) possui três dimensões de análise: o Registro da Ação (relacionado ao planejamento da atividade em consonância com as Diretrizes para a Extensão), a Avaliação da Submissão (referente à potencial geração de produtos, aos planos de trabalho e à experiência do coordenador da atividade) e o Acompanhamento da Ação (alusivo ao desempenho da atividade e à interação entre docentes, discentes e comunidade).

O Projeto ReFormar, apresentado e discutido nas seções seguintes, busca atender aos critérios especificados na primeira dimensão, o Registro da Ação, o que reforça a premissa dessa atividade de atender às Diretrizes para a Extensão Universitária instituídas em 2018.

\section{O PROJETO REFORMAR}

O Programa de Educação Tutorial (PET) visa fomentar a formação técnica e cidadã de estudantes de graduação, estejam estes envolvidos direta ou indiretamente com o programa. Para atingir esse objetivo geral, os grupos PET desenvolvem atividades variadas que aproximam os estudantes de seu futuro ambiente profissional articulando os pilares da tríade acadêmica (BRASIL, 2006).

O PET Civil - UFJF, por sua vez, já publicou resultados de atividades de ensino (e. g. Carvalho, F. et al. (2020), Carvalho, Y. et al. (2020), Oliveira et al. (2017) e Velasco et al. (2018)), pesquisa (e. g. Carvalho, Y. (2020), Castro et al. (2019), Oliveira, Almeida e Carvalho (2019); Ribeiro e Riccio Filho (2019) Valle, Parreiras e Bernardes (2018) e Velasco e Barbosa (2019)) e extensão (e.g. Bernardes et al. (2020) Valle et al. (2017)). Em reuniões internas, no entanto, o grupo reconheceu a predominância de atividades de ensino e pesquisa em detrimento de atividades de extensão. Nesse contexto, o PET Civil - UFJF se dedicou a conceber um novo projeto (etapas da atividade ilustradas na Figura 1) que, fundamentado nas Diretrizes para a Extensão na Educação Superior Brasileira (BRASIL, 2018), proporcione a melhoria acadêmica do curso de Engenharia Civil da Universidade Federal de Juiz de Fora. 
Figura 1 - Etapas para implementação do Projeto ReFormar.

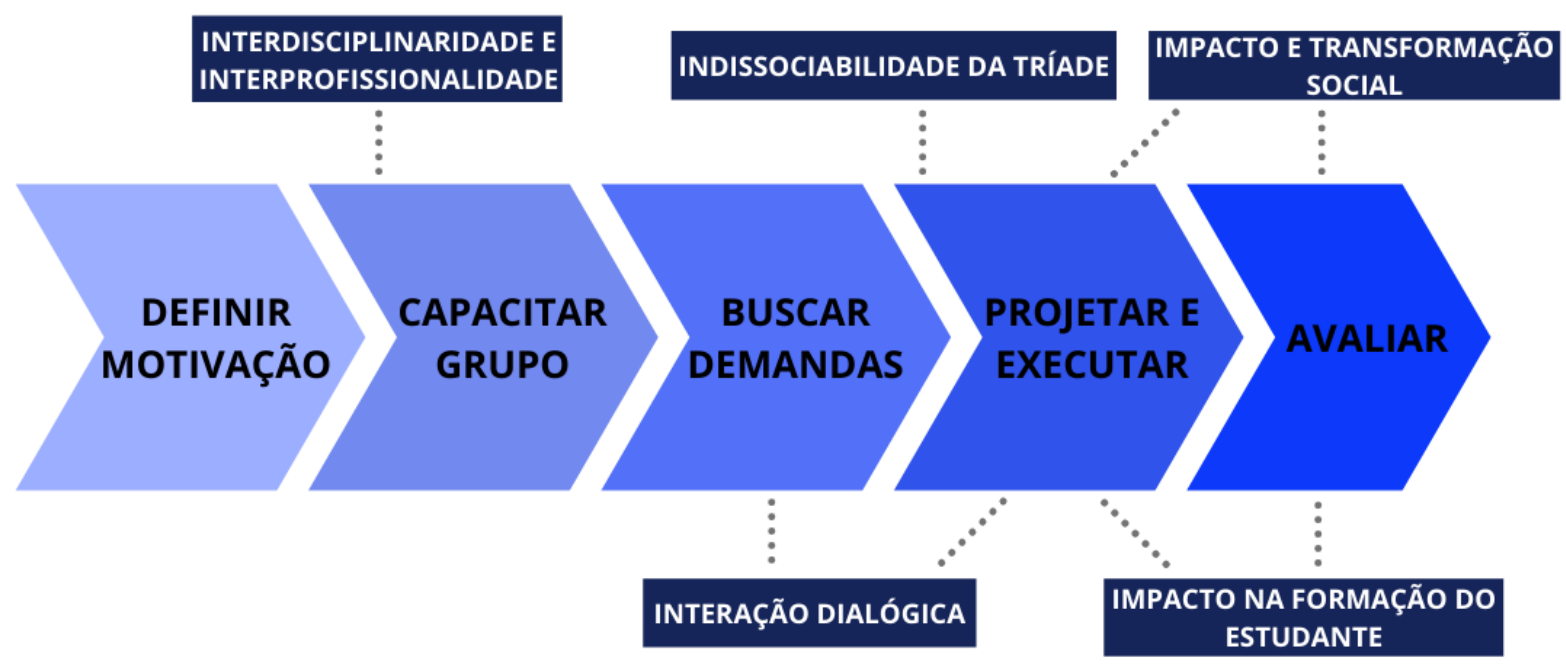

Fonte: os autores (2020).

\subsection{Definir a motivação}

A mobilização em prol de uma nova atividade de extensão começou em fevereiro de 2020. Após observar como outros projetos da universidade atuavam, foi proposto, inicialmente, que o PET Civil - UFJF identificasse demandas passíveis de serem solucionadas com pequenos projetos de Engenharia em Juiz de Fora - MG e em municípios vizinhos. Assim, esperava-se contribuir, também, para a formação dos discentes envolvidos com a atividade, visto que o projeto e a implementação das soluções promoveriam um ambiente de aprendizagem ativa consonante com as DCNs de Engenharia.

No entanto, a pandemia de COVID-19 impediu que o grupo mobilizasse esforços para implementar a ideia na prática. A fim de reestruturar a atividade, uma comissão foi composta com membros do PET Civil - UFJF para identificar o que poderia ser desenvolvido remotamente e para reunir documentação e bibliografia pertinentes sobre Extensão Universitária. Ao estudar as diretrizes propostas na PNEU (FORPROEX, 2012) e na Resolução no 7, de 18 dezembro de 2018 (BRASIL, 2018), o grupo reconheceu que a proposta inicial de prestação de serviços não previa uma interação dialógica com a comunidade, negando a ideia da Extensão como uma via de mão dupla.

Nesse contexto, foi concebido o Projeto ReFormar. A nova atividade tem por objetivo oferecer auxílio técnico de Engenharia Civil a instituições sociais ou públicas a fim de construir junto à sociedade novos conhecimentos e produtos, promovendo uma maior interação entre a comunidade e a universidade. A palavra "reformar", que carrega o sentido de reconstituir, melhorar a forma de algo, foi estilizada como ReFormar a fim de atentar para a nova formação técnica e cidadã que será proporcionada pela atividade.

\subsection{Capacitar o grupo}

A fim de determinar a dinâmica de trabalho para o projeto, o PET Civil - UFJF identificou os serviços de Engenharia ofertados por outros segmentos universitários da mesma IES. Além disso, o grupo começou a listar atores externos à universidade (como empresas, escritórios e profissionais autônomos do ramo da Construção Civil) que poderiam colaborar com a atividade. Ao identificar as especialidades de cada possível colaborador, o contato em prol de parcerias é facilitado - sejam tais parcerias voltadas para a atuação conjunta em algum caso específico ou para a capacitação dos membros do PET Civil - UFJF. Assim, permite-se que os discentes envolvidos na atividade vivenciem 
uma aprendizagem interdisciplinar e interprofissional, colaborando para uma formação técnica que não seria vivenciada em uma sala de aula tradicional.

A busca por capacitações, por sua vez, não se limitará a conhecimentos técnicos de Engenharia. O PET Civil - UFJF também estudará questões de cunho social que permitam aos estudantes envolvidos no projeto refletir sobre as relações entre Engenharia e sociedade e estabelecer diálogos construtivos com as comunidades com as quais firmarem contato.

\subsection{Identificar demandas junto à sociedade}

O levantamento de demandas junto à população dar-se-á após o PET Civil - UFJF se apropriar dos conhecimentos necessários para coleta de dados, como práticas para conduzir grupos focais e entrevistas individuais - prezando sempre por abordagens que não caracterizem invasão cultural. Dessa forma, espera-se desenvolver um trabalho dialógico que permita tanto ao grupo quanto à comunidade externa à IES compreender o papel de cada um na transformação da realidade em que se inserem.

\subsection{Conceber, projetar e implementar a solução}

A ação sobre a problemática levantada dar-se-á a partir da articulação entre os conhecimentos técnicos mobilizados pelo PET Civil - UFJF e os conhecimentos populares pertencentes à comunidade alvo da atividade de extensão. Seguindo as recomendações de Krick (2006), as informações coletadas e discutidas em conjunto com a comunidade permitirão uma formulação ampla do problema, o qual será analisado considerando aspectos técnicos (o que deverá ser feito, quando, por quem etc.), educacionais (resultados de aprendizagem almejados com a atividade) e sociais (impacto que o desenrolar da atividade causará na comunidade) e respeitando as restrições às quais o problema está sujeito (como restrições econômicas, temporais, de mão de obra etc.). Logo, ao projetar soluções para o problema, deve-se considerar não apenas o produto final que será entregue, mas também os meios pelos quais o problema será resolvido e como esses meios afetarão à comunidade.

A fim de atender às questões propostas por Kienetz, Vieira e Visentini (2020), o PET Civil - UFJF e a comunidade discutirão como cada qual contribuirá para as etapas de planejamento, execução e avaliação da atividade - reforçando, assim, a interação dialógica preconizada no marco legal de Extensão (BRASIL, 2018). A fim de reforçar o caráter interdisciplinar e interprofissional, o grupo PET buscará firmar parcerias junto a outros segmentos universitários e representantes da sociedade civil tanto para capacitar os envolvidos com o projeto quanto para fornecer materiais e apoios diversos. Desse modo, ao combinar conhecimentos advindos da Universidade e da comunidade, é possível produzir novos conhecimentos que transformam tanto os estudantes quanto a sociedade envolvida com o projeto - atendendo à premissa da indissociabilidade da tríade acadêmica.

\subsection{Avaliar o desenvolvimento da atividade}

Pretende-se avaliar o Projeto ReFormar seguindo três critérios principais: o desenvolvimento da atividade, o impacto na formação discente e o impacto na comunidade. Ao avaliar o desenvolvimento da atividade, o PET Civil - UFJF coletará impressões qualitativas e quantitativas de membros do programa, estudantes e representantes da comunidade a fim de identificar pontos positivos e sugestões de melhoria para projetos futuros. Essa avaliação ocorrerá tanto ao final da atividade quanto durante 0 desenvolvimento da mesma, o que possibilita aprimorar o projeto a cada iteração. $O$ 
impacto na formação discente será avaliado juntamente aos estudantes que participaram do projeto (sendo eles membros do PET ou não). Considerando os resultados de aprendizagem previamente definidos, os discentes serão convidados a pontuar o grau de desenvolvimento das competências propostas e a comentar a vivência da atividade, fornecendo dados para melhorias futuras. Por fim, a comunidade externa também será convidada a avaliar a atividade, contribuindo com suas impressões acerca do impacto do projeto em sua realidade, de sua satisfação com o resultado final e da experiência de trabalho com a Universidade.

\section{CONSIDERAÇÕES FINAIS}

O objetivo deste artigo foi apresentar o embasamento teórico e as etapas idealizadas para o Projeto Reformar - atividade de extensão do PET Civil - UFJF que visa promover o compartilhamento e a construção conjunta de conhecimentos de forma dialógica entre a Universidade e a comunidade alvo do projeto.

Os estudantes envolvidos no projeto terão a possibilidade de vivenciar os conhecimentos adquiridos na graduação e, assim, desenvolver competências por meio da aprendizagem ativa - tanto técnicas quanto sociais. A comunidade, por sua vez, construirá o conhecimento junto aos estudantes e atuará ativamente para a transformação de sua realidade. Dessa forma, será possível estabelecer uma simbiose entre Universidade e sociedade, promovendo a democratização do conhecimento técnico de Engenharia e combinando-o ao saber popular da comunidade.

Por fim, mesmo que a pandemia de COVID-19 tenha impossibilitado dar início à atividade em um contexto real, reforça-se que a discussão teórica que fundamenta o Projeto ReFormar poderá contribuir para o desenvolvimento de ações de mesma natureza em outras IES, uma vez que essa proposta de atividade apresenta possibilidades para o cumprimento das Diretrizes para a Extensão na Educação Superior Brasileira.

\section{REFERÊNCIAS}

ALÁYON, N. O assistencialismo na política social e no Serviço Social. In: Assistência e assistencialismo: controle dos pobres ou erradicação da pobreza?. São Paulo: Cortez, 1992.

BAZZO, W. A.; PEREIRA, L. T. V. Introdução à Engenharia: conceitos, ferramentas e comportamentos. Florianópolis: Editora da UFSC, 2006. 270 p.

BRASIL. Art. 207. In: Constituição da República Federativa do Brasil. Brasília, Senado Federal, 1988. Disponível em: <https://www.senado.leg.br/atividade/ const/con1988/con1988_18.02.2016/art_207_.asp>. Acesso em 19 abr. 2021.

BRASIL. Ministério da Educação, Conselho Nacional de Educação, Câmara de Educação Superior. Resolução CNE/CES no 1, de 19 de dezembro de 2020. Brasília: Diário Oficial da União, 2020. p. 64.

BRASIL. Ministério da Educação, Conselho Nacional de Educação, Câmara de Educação Superior. Resolução no 2, de 24 de abril de 2019. Brasília: Diário Oficial da União, 2019. p. 43-44. 
BRASIL. Ministério da Educação, Conselho Nacional de Educação, Câmara de Educação Superior. Resolução no 7, de 18 de dezembro de 2018. Brasília: Diário Oficial da União, 2018. p. 49-50.

BRASIL. Ministério da Educação, Secretaria de Educação Superior, Departamento de Modernização e Programas da Educação Superior, Coordenação Geral de Relações Acadêmicas de Graduação. Programa de Educação Tutorial - PET: Manual de Orientações Básicas. Brasília: [s.n.], 2006. 25 p.

BERNARDES, R. R. et al. Inovação em tempos de crise: capacitação a distância. In: XLVIII Congresso Brasileiro de Educação em Engenharia e III Simpósio Internacional de Educação em Engenharia da ABENGE, 2020 [evento on-line]. Anais... [S.I.]: ABENGE, 2020. p. 1-8. doi: 10.37702/COBENGE.2020.2902.

CARVALHO, F. A. et al. Aprendizagem ativa a partir de competições: Concurso de Taludes. In: XLVIII Congresso Brasileiro de Educação em Engenharia e III Simpósio Internacional de Educação em Engenharia da ABENGE, 2020 [evento on-line]. Anais... [S.I.]: ABENGE, 2020. p. 1-8. doi: 10.37702/COBENGE.2020.2913.

CARVALHO Y. M. et al. Semana da Evolução . In: XXV Encontro Nacional dos Grupos PET, 2020 [evento on-line]. Anais... [S.I.]: UFPR, 2020. p. 1-5.

CARVALHO, Y. M.; PINTO, V. G. Panorama histórico do combate à umidade na Construção Civil: das paredes de adobe à aurora do Terceiro Milênio. Revista Thema, v. 17, p. 45-56, 2020. doi: 10.15536/thema.V17.2020.45-56.1530.

CASTRO, C. M. et al. Parceria Universidade-Prefeitura: um estudo do tráfego de veículos em Juiz de Fora. In: VI Congresso Nacional dos Grupos PET de Engenharia Civil, 2019, Porto Alegre - RS. Anais... Campinas: Galoá, 2019. p. 1-5. doi: 10.17648/conpet-2019107561.

FORPROEX - FÓRUM DE PRÓ-REITORES DE EXTENSÃO DAS INSTITUIÇÕES PÚBLICAS DE EDUCAÇÃO SUPERIOR BRASILEIRAS. Política Nacional de Extensão Universitária. Florianópolis: Imprensa Universitária - IU, set. 2015. 69 p.

FREIRE, P. Educação ou comunicação?. 7ª ed. Rio de Janeiro: Paz e Terra, 1983. 93 p.

KIENETZ, T. B.; VIEIRA, K. M.; VISENTINI, M. S. Extensão Universitária: Avaliar para evoluir. Teoria e Prática em Administração, v. 10, n. 1, p. 111-118, 2020. doi:10.21714/2238-104X2020v10i1-48519.

KRICK, E. V. Introducción a la ingeniería y al diseño en la ingeniería. México: Limusa, 2006. 240 p.

MOREIRA, J. da S. Extensão universitária: entre o assistencialismo e o compromisso com o povo. Fragmentos de Cultura, Goiânia, v. 24, especial, p. 25-30, dez. 2014. 
OLIVEIRA, M. A. et al. Concurso Mola In: IV Congresso Nacional dos Grupos PET de Engenharia Civil, 2017, Fortaleza - CE. Anais... Campinas: Galoá, 2017. p. 1-5. doi: 10.17648/conpet-2017-60254.

OLIVEIRA, T. F.; ALMEIDA, J. R. de; CARVALHO, N. G. Contaminação do Solo por Metais Pesados no Entorno de um Lixão Desativado. In: IX Congresso Brasileiro de Geotecnia Ambiental (REGEO 2019) e VIII Congresso Brasileiro de Geossintéticos (Geossintéticos 2019), 2019, São Carlos - SP. Anais... São Carlos: IGS-Brasil/ABMS, 2019. p. 579-586.

RIBEIRO, E. F.; RICCIO FILHO, M. V. Construção de um pluviador de areia para moldagem de corpos de prova com diferentes densidades e índices de vazios. In: VI Congresso de Engenharia Civil (CONENGE), 2019, Juiz de Fora - MG. Anais... Juiz de Fora: Templo, 2019.

VALLE, A. de A. et al. III Concurso de Pontes de Papel para as Escolas Públicas de Juiz de Fora. In: XLV Congresso Brasileiro de Educação em Engenharia, 2017, Joinville - SC. Anais... Joinville: ABENGE, 2017. p. 1-9.

VALLE, A. de A.; PARREIRAS, E. A.; BERNARDES, M. V. Softwares para projeto, análise e simulação de estruturas de Engenharia Civil e Arquitetura. In: V Congresso Nacional dos Grupos PET de Engenharia Civil, 2018, Ouro Preto - MG. Anais... Campinas: Galoá, 2018. p. 1-5. doi: 10.17648/conpet-2018-84810.

VELASCO, L. F.; BARBOSA, M. T. Postos de saúde sustentáveis: sugestões de adequações a serem feitas nos serviços de manutenção. In: VI Congresso de Engenharia Civil (CONENGE), 2019, Juiz de Fora - MG. Anais... Juiz de Fora: Templo, 2019. p. 77-89.

\title{
PROJETO REFORMAR: EXTENSION IN CIVIL ENGINEERING BASED ON THE BRAZILIAN GUIDELINES FOR EXTENSION IN HIGHER EDUCATION
}

\begin{abstract}
According to the Brazilian Guidelines for Extension in Higher Education, extension activities must transform University and society through a dialogical interaction that enables sharing and building knowledge together. However, it is hard to perform extension activities that stimulate competencies development by students and positively impact society. To cope with this, the Educational Tutorial Program of the Civil Engineering undergraduate course of the Federal University of Juiz de Fora (PET Civil - UFJF) elaborated an extension activity based on the Brazilian Guidelines for Extension in Higher Education named Projeto ReFormar. The project aims to offer Civil Engineering technical aid to social or public institutions to create new knowledge and products with the target community. This paper synthesizes the rationale for planning the activity, the proposed steps for its execution, and the expected results of its implementation. Therefore, we hope to attend the Brazilian Guidelines for University Extension and incentive similar extension activities in other Higher Education Institutions.
\end{abstract}

Keywords: Engineering and society. Social transformation. Academic triad. Assistentialism. 